\title{
Caveolae protect endothelial cells from membrane rupture during increased cardiac output
}

\author{
Jade P.X. Cheng, ' Carolina Mendoza-Topaz, ' Gillian Howard,' Jessica Chadwick,' Elena Shvets,' \\ Andrew S. Cowburn, ${ }^{3}$ Benjamin J. Dunmore, ${ }^{2}$ Alexi Crosby, ${ }^{2}$ Nicholas W. Morrell, ${ }^{2}$ and Benjamin J. Nichols ${ }^{1}$ \\ 1Medical Research Council, Laboratory of Molecular Biology, ${ }^{2}$ Department of Medicine, and ${ }^{3}$ Department of Physiology, University of Cambridge, Cambridge CB2 ITN, UK
}

Caveolae are strikingly abundant in endothelial cells, yet the physiological functions of caveolae in endothelium and other tissues remain incompletely understood. Previous studies suggest a mechanoprotective role, but whether this is relevant under the mechanical forces experienced by endothelial cells in vivo is unclear. In this study we have sought to determine whether endothelial caveolae disassemble under increased hemodynamic forces, and whether caveolae help prevent acute rupture of the plasma membrane under these conditions. Experiments in cultured cells established biochemical assays for disassembly of caveolar protein complexes, and assays for acute loss of plasma membrane integrity. In vivo, we demonstrate that caveolae in endothelial cells of the lung and cardiac muscle disassemble in response to acute increases in cardiac output. Electron microscopy and two-photon imaging reveal that the plasma membrane of microvascular endothelial cells in caveolin $1^{-/-}$mice is much more susceptible to acute rupture when cardiac output is increased. These data imply that mechanoprotection through disassembly of caveolae is important for endothelial function in vivo.

\section{Introduction}

Caveolae, flask-shaped invaginations of the plasma membrane, are highly abundant in endothelial cells, and are therefore likely to play an important role in endothelial cell biology (Tse and Stan, 2010). Defining precisely how caveolae function in the endothelium and elsewhere has proved challenging, but phenotypes of mice lacking genes essential for the biogenesis of caveolae provide evidence that caveolae play an important role in the cardiovascular system. These mice have alterations in the permeability of continuous endothelium (Schubert et al., 2002); impaired angiogenesis in tumor models (Chang et al., 2009; Siddiqui et al., 2011); are prone to pulmonary hypertension and dilated cardiomyopathy (Zhao et al., 2002; Cruz et al., 2012); and are highly exercise intolerant (Drab et al., 2001). Humans with mutations in the same genes also exhibit cardiac arrhythmias and pulmonary hypertension, and have enlarged blood vessels (Rajab et al., 2010; Austin et al., 2012). Caveolae-deficient mice and humans have metabolic phenotypes consistent with a role for caveolae in adipocytes (Pilch and Liu, 2011) and muscular dystrophy (Galbiati et al., 2001a,b; Woodman et al., 2004; Parker et al., 2007; Ardissone et al., 2013). Adipocytes and muscle cells are also cells where caveolae are abundant; hence, there is a correlation between abundance of caveolae and their importance for cell function.

Caveolae are formed by large complexes of caveolin and cavin proteins (Hill et al., 2008; Hayer et al., 2010; Ludwig et al., 2013; Gambin et al., 2014). Caveolins are multiply acylated, embedded in the inner leaflet of the plasma membrane,

Correspondence to Benjamin J. Nichols: ben@mrc-Imb.cam.ac.uk and oligomerize to form the most membrane-proximal component of the caveolar coat complex. Cavins are soluble proteins that are recruited to caveolins after biosynthetic delivery of the latter to the plasma membrane. Caveolin 1 and cavin 1 are both essential for formation of caveolae; caveolin $1^{-/-}$mice lack caveolae in all cell types except for striated muscle and cavin $1^{-/-}$ mice lack caveolae in all tissues (Drab et al., 2001; Razani et al., 2001; Liu et al., 2008). Caveolin 1, caveolin 2, cavin 1, and the additional cavins 2 and 3 , can all be purified from cells as a single $80 \mathrm{~S}$ caveolar coat complex after chemical cross-linking (Ludwig et al., 2013).

Possible functions for endothelial caveolae include control of the endothelial nitric oxide synthase (García-Cardeña et al., 1996; Siddiqui et al., 2011); transport of ligands and solutes across the endothelial cell as transcytotic vesicles (Oh et al., 2007; Predescu et al., 2007); mechanotransduction (Albinsson et al., 2008; Joshi et al., 2012); and further signaling processes (Parton and Simons, 2007; Collins et al., 2012).

Recent experiments in cultured cells have revived the idea that caveolae may have a simple mechanoprotective role (Dulhunty and Franzini-Armstrong, 1975; Sinha et al., 2011; Parton and del Pozo, 2013). In this model, caveolae act as membrane convolutions that can flatten in response to forces within the membrane, thereby buffering such forces and reducing the

(C) 2015 Cheng et al. This article is distributed under the terms of an AttributionNoncommercial-Share Alike-No Mirror Sites license for the first six months after the publication date (see http://www.rupress.org/terms). After six months it is available under a Creative Commons License (Attribution-Noncommercial-Share Alike 3.0 Unported license, as described at http://creativecommons.org/licenses/by-nc-sa/3.0/). 
chance of critical membrane rupture or loss of cell-cell contact (Parton and del Pozo, 2013). The model is attractive because it provides a good explanation for the abundance of caveolae in some cell types. Both stretch-dependent changes in the abundance of caveolae in isolated muscle fibers and pressure-dependent changes in abundance of endothelial caveolar vesicles have been observed (Dulhunty and Franzini-Armstrong, 1975; Lee and Schmid-Schönbein, 1995); however, direct in vivo evidence that caveolae do indeed disassemble or flatten in response to physiological forces, and thereby protect the plasma membrane from disruption by mechanical stress, has been lacking. The situation is complicated by data suggesting that endocytosis of caveolae occurs in response to plasma membrane damage (Corrotte et al., 2013; Andrews et al., 2014; Shvets et al., 2015). Both endocytosis and flattening of caveolae may cause changes in the abundance of these structures at the plasma membrane, thus it is crucial to develop assays for disassembly of caveolae that do not rely solely on morphological criteria.

The goal of this study was to address the potential mechanoprotective function of caveolae in endothelial cells in vivo. We designed experiments to test the two key aspects of the hypothesis that disassembly and flattening out of caveolae buffers mechanical forces within the plasma membrane (Sinha et al., 2011). First, we asked whether disassembly of caveolae can be detected in intact tissue under physiological conditions. We used tissue culture cell experiments to characterize both biochemical and electron microscopy assays for caveolar disassembly and applied these assays to mice where cardiac output, and hence hemodynamic force on endothelial cells, is increased. Additional experiments rule out endocytosis as an alternative explanation for changes in caveolar abundance. Second, we established an assay for acute damage to the plasma membrane based on membrane-impermeant nuclear dyes, validated this assay in cell culture experiments comparing control and caveolin $1^{-/-}$cells, and applied the assay to mice under conditions of increased cardiac output.

\section{Results and discussion}

\section{Biochemical assays for disassembly of caveolae under mechanical force}

Cells of the endothelium-derived cell line bEnd5 were mechanically stressed by exposure to hypo-osmotic medium, and by cycles of repeated stretching after growth on polydimethylsiloxane, a deformable substrate. We quantified the effect of these treatments on the abundance of morphologically defined caveolae using electron microscopy of complete perimeters of multiple individual cells (Fig. S1, A and B; Hansen et al., 2013). As predicted, both treatments rapidly and significantly reduced the number of morphological caveolae (Sinha et al., 2011).

If the loss of caveolae (Fig. S1) were because of disassembly of caveolar coat complexes, then one would predict that this would release soluble caveolar proteins such as cavin 1 from the membrane into the cytoplasm (Hayer et al., 2010; Sinha et al., 2011; Gambin et al., 2014). Western blotting of cytosolic fractions after ultracentrifugation to pellet membranes showed that both hypo-osmotic medium and stretching on a deformable substrate clearly caused a time-dependent increase in the cytosolic pool of cavin 1 (Fig. 1, A and B). Caveolin 1 remained in the membrane fraction, and the total amount of cavin 1 present in the cell did not change during the experiment (Fig. 1, C and D).
Interpretation of cytosolic accumulation of cavin 1 as direct evidence for disassembly of caveolae is a central part of our conclusions; therefore, we performed additional experiments to confirm this. The cavin 1 released into the cytosol under hypo-osmotic conditions was present in two peaks on sucrose velocity gradients. The higher molecular weight peak (fraction 8 ) had the same size as the basal amount of cytosolic cavin 1 detected normally. Crucially, the second peak, comprising smaller complexes (fraction 4), was not observed under isotonic conditions (Fig. 1 E). Therefore, large cavin 1-containing complexes disassemble under mechanical stress induced by hypotonic conditions (Gambin et al., 2014).

Essentially all of the cavin and caveolin proteins present in caveolae can be purified as a single 80S complex after chemical cross-linking (Ludwig et al., 2013). If caveolae disassemble, then this complex should be lost. Incubation of cells under hypo-osmotic conditions for $10 \mathrm{~min}$ did indeed result in loss of the cross-linked 80S complex because caveolin 1 and cavin 1 no longer cofractionated in high molecular weight fractions on sucrose gradients and instead accumulated in much smaller complexes (Fig. 1, F and G). This provides direct evidence for disassembly of the caveolar coat complex, and confirms that the accumulation of cavin 1 in the cytosol reported in Fig. 1 corresponds to disassembly of caveolae.

It was possible that changes in the abundance of morphologically defined caveolae observed by electron microscopy could reflect budding from the plasma membrane. Accordingly, we performed endocytosis assays using total surface protein biotinylation to label all endosomes in bEnd5 cells (Bitsikas et al., 2014). These experiments were performed using both hypo-osmotic buffer and stretching after growth on a deformable substrate. In hypo-osmotic conditions the extent to which caveolin 1 colocalized with cavin 1 was markedly reduced, consistent with disassembly of caveolae, and under these conditions endocytosis was blocked (Fig. 2, A and B). Mechanical stretching by $20 \%$ with a frequency of $1 \mathrm{~Hz}$ reduced total endocytosis (Fig. 2 D), and colocalization between cavin 1 and caveolin 1 was also reduced (Fig. 2 C). Quantification, using a pixel-mask approach (Shvets et al., 2015), revealed that the amount of caveolin 1 in endocytic structures did not change significantly during mechanical stretching (Fig. 2 E). We conclude that loss of morphologically defined caveolae from the plasma membrane is, therefore, indeed because of disassembly of caveolae.

Disassembly of endothelial caveolae in vivo To ascertain whether the forces generated by increased blood flow acutely trigger disassembly of endothelial caveolae we used the $\beta 1$-adrenoreceptor agonist dobutamine. This causes increased heart contractility and output and is used clinically to mimic effects of exercise (Marcovitz and Armstrong, 1991). Pulse plethysmography showed that dobutamine caused a pronounced elevation of both pulse rate and pulse distension $1 \mathrm{~min}$ after i.p. injection, and both parameters reached levels seen in exercising mice (Fig. S2 A; Desai et al., 1997). Tissues from control and caveolin $1^{-/-}$mice treated for $1 \mathrm{~min}$ with dobutamine were very rapidly dissected and snap frozen. After homogenization and cell lysis the soluble and membrane fractions from each tissue were isolated by centrifugation, and the presence of cavin 1 was assayed by Western blotting (see Fig. 4 A and Fig. S2 B). In both the heart and lung dobutamine caused an increase in the amount of soluble cavin 1 (Fig. 3, A and B; controls in Fig. S2 B). This was not the case in abdominal muscle. 
A

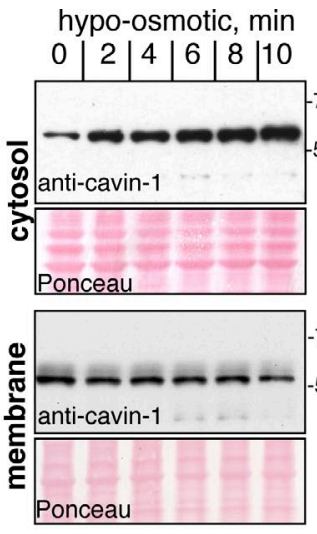

D

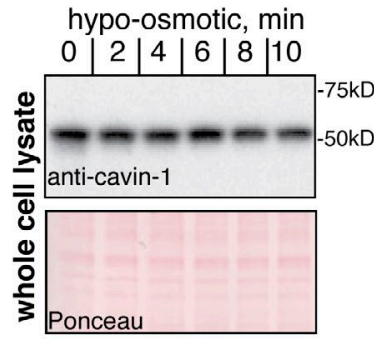

B stretch, $\min$

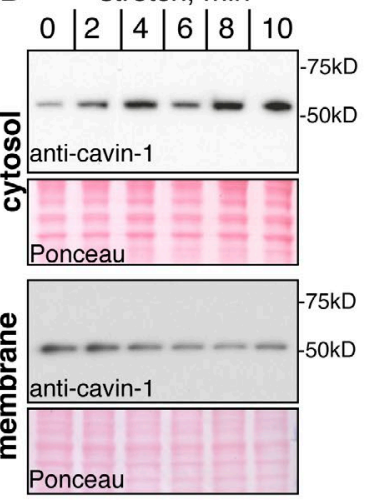

E

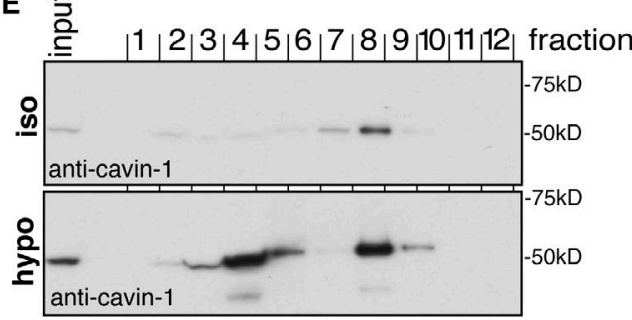

$\mathbf{F}$

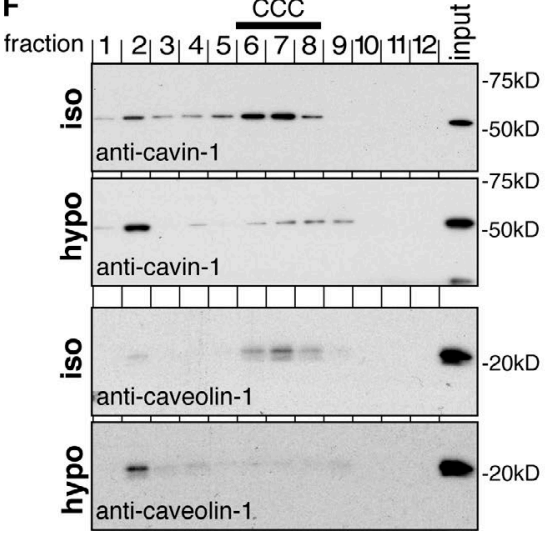

C hypo-osmotic, $\min$

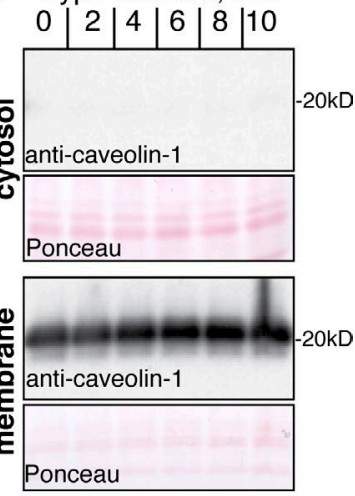

$-20 k D$

(1)
and membrane fractions from cells lysed after different times in hypo-osmotic medium. Ponceau staining of the relevant areas of the blot membrane is included to demonstrate equal loading. (B) Immunoblot analyses of cavin 1 in cytosolic and membrane fractions from cells lysed after different times of mechanical stretching, the deformable substrate on which the cells were grown was stretched by $20 \%$, repeated at $1 \mathrm{~Hz}$. (C) Immunoblot analyses of caveolin 1 in cytosolic and membrane fractions from cells lysed after different times in hypo-osmotic medium. Ponceau staining of the relevant areas of the blot membrane is included to demonstrate equal loading. (D) Immunoblot analyses of cavin 1 whole cell lysates after different times in hypo-osmotic medium. (E) Cytosolic fractions from cells incubated for $10 \mathrm{~min}$ in isotonic (iso) or hypotonic (hypo) buffer were applied to a 10-40\% sucrose velocity gradient, and analyzed by Western blotting of fractions 1-12. (F) Cross-linked and detergent-solubilized $11 \%$ Triton X-100/1\% octyl-glucoside) cell extracts were fractionated by velocity centrifugation (10-40\% sucrose), followed by Western blotting of fractions 1-12.

Gradients were prepared from cells incubated in isotonic or hypotonic buffer for $10 \mathrm{~min}$. The high molecular weight peak of caveolin 1 and cavin 1 corresponding to the caveolar coat complex (CCC) in isotonic medium is indicated. (G) Quantification of the distribution of cavin 1 and caveolin 1 in velocity gradients as shown in F. Protein amounts normalized such that area under each curve equal to 1 was determined by densitometry of Western blots.
The data suggest that caveolae disassemble in heart and lung in response to dobutamine-induced increases in cardiac output.

Electron microscopy was used to confirm that morphologically defined caveolae become less abundant in endothelial cells in response to dobutamine. The time taken for dissection and fixation of tissue and for heterogeneity in caveolar abundance between different sections of endothelium from the same tissue is a potentially confounding factor in this type of experiment. We focused on the heart and the lung, tissues that can be dissected rapidly, and where the biochemical experiments reported previously suggested that an effect would be found. In both tissues electron microscopy of microvessels revealed that there are considerable stretches of plasma membrane devoid of caveolae in the dobutamine-treated samples, although caveolae are clearly still present (Fig. 3, C and D). Quantification of the number of caveolae, defined as distinctive omega shapes open at the luminal face of the endothelial cell, in complete reconstructions of microvessel perimeters from control and dobutamine-treated mice revealed a reduction in response to the drug (Fig. 3, E and F). Therefore, endothelial caveolae are indeed likely to disassemble in response to increased cardiac output.

\section{Caveolae prevent rupture of endothelial plasma membranes under physiological hemodynamic force}

If disassembly of caveolae does protect cells from plasma membrane disruption under mechanical stress, then one would predict that, in the absence of caveolae, cells might be more susceptible to acute rupture of the plasma membrane. In order assay acute membrane rupture we used membrane-impermeant, nucleicacid-binding dyes, which will confer bright staining of the nucleus only when integrity of the plasma membrane is lost (Jones and Singer, 2001). Establishing the assay in tissue culture cells, control and caveolin $1^{-/-}$mouse embryonic fibroblasts were exposed to hypo-osmotic medium or mechanical stretching in the presence of SYTOX Green, as well as fluorescent wheat germ agglutinin to track the location and morphology of individual cells. After 4 min in hypo-osmotic buffer, prominent nuclear staining was observed in many of the caveolin $1^{-/-}$cells (Fig. S3 A). SYTOX Green staining was quantified using flow cytometry (Fig. S3 B). Again, there was clearly significantly more staining in the caveolin $1^{-/-}$cells. Mechanical stretching experiments yielded essentially the same result because there were significantly more SYT OX Green-positive nuclei in caveolin $1^{-1-}$ cells when stretched 

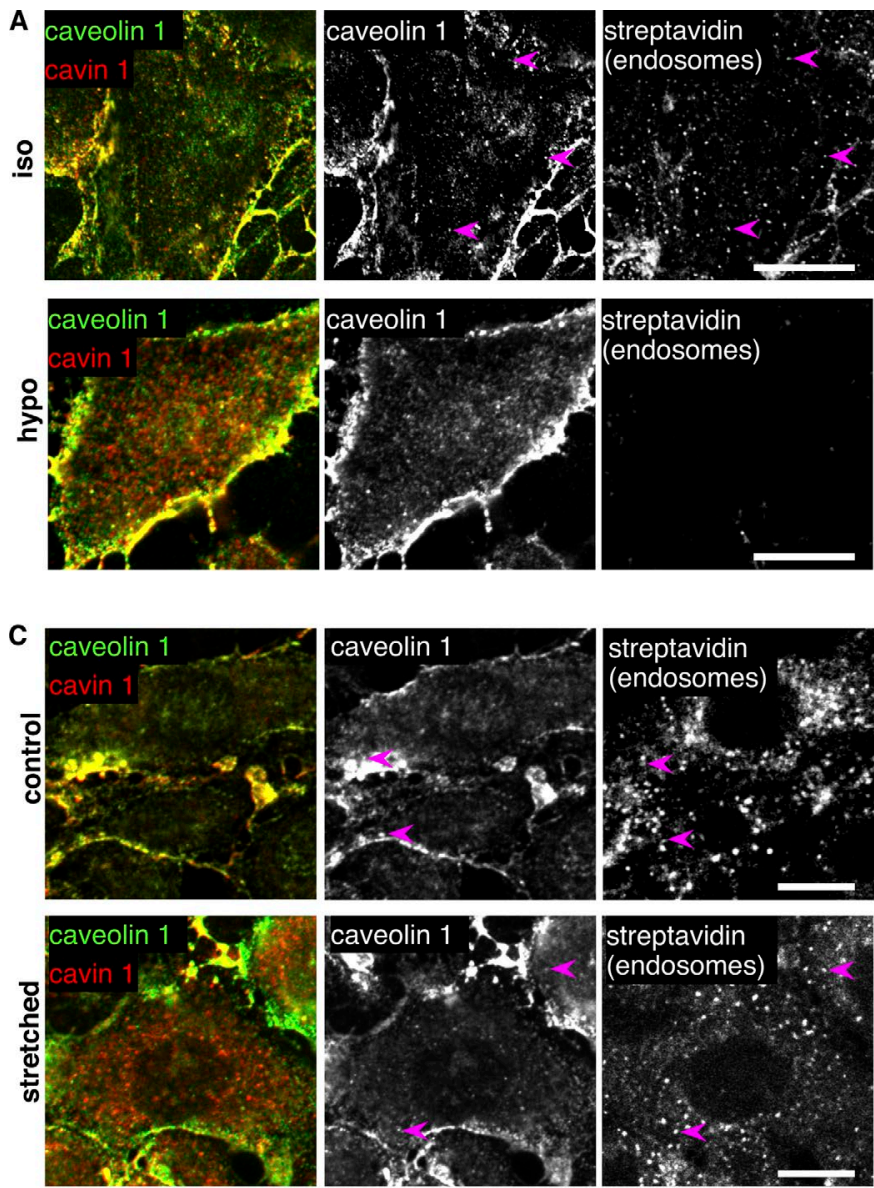

streptavidin
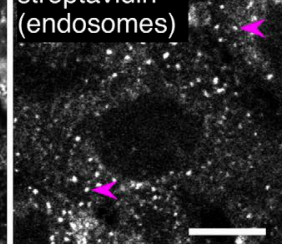

$\mathbf{E}$ $\%$ caveolin 1 in endosomes,

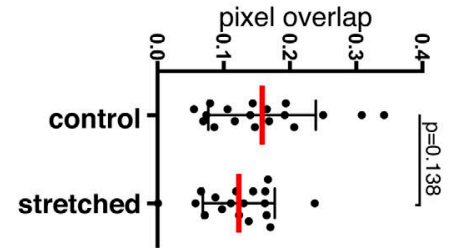

as in previous experiments than in the controls. In this case, quantification was performed by confocal imaging (Fig. S3 C). These experiments show that staining with membraneimpermeant nuclear dyes can be used to assay plasma membrane disruption, and that caveolae do indeed protect cells from rupture of the plasma membrane during increased membrane tension.

Intravenous injection of FITC-albumin and propidium iodide (a membrane-impermeant nuclear dye analogous to SYT OX Green) provided a way to image blood vessels in intact tissue, and to label nuclei only where the plasma membrane is damaged. Two-photon imaging was performed in cardiac muscle from control and caveolin $1^{-/-}$mice, with injection of dobutamine to acutely increase cardiac output (Fig. 4 A and Fig. S2 A). Vessels in caveolin $1^{-/-}$animals were slightly less well-defined relative to signal from tissue, possibly reflecting an increase in permeability of the endothelium (Schubert et al., 2002; Hansen et al., 2013). Loss of plasma membrane integrity in endothelial cells was indicated by nuclear staining with propidium iodide that colocalized with blood vessels highlighted with FITC-albumin. Although there was little or no membrane damage in controls, damaged endothelial cells were readily observed and abundant in the caveolin $1^{-/-}$, dobuta-

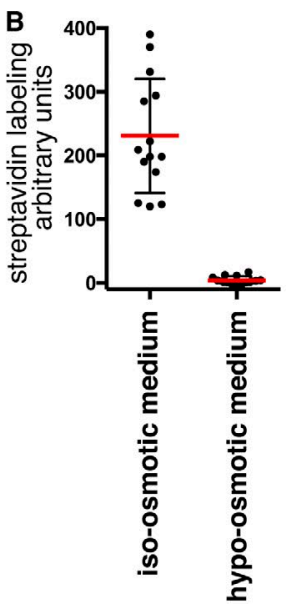

Figure 2. Mechanical force does not induce endocytosis of caveolae. (A) Surface biotinylation using reducible sulfo-NHS-SS-biotin was followed by incubation for $10 \mathrm{~min}$ in isotonic or hypotonic medium at $37^{\circ} \mathrm{C}$ as shown. After MESNa treatment to remove noninternalized biotin, cells were fixed and labeled with antibodies against caveolin 1 and cavin 1, and with fluorescent streptavidin. Arrows highlight limited presence of caveolin 1 in endocytic structures. Note that the streptavidin channel is omitted from the red/green overlay images. Bars, $10 \mu \mathrm{m}$. (B) Quantification of biotin uptake after surface labeling with sulfo-NHS-SS-biotin and MESNa treatment. Mean fluorescence in tensity of multiple cell regions after subtraction of background signal is shown. (C) Cells were labeled with sulfo-NHS-SS-biotin, followed by incubation for $10 \mathrm{~min}$ at $37^{\circ} \mathrm{C}$, whereas the deformable substrate on which the cells were grown was stretched by $20 \%$, repeated at 1 $\mathrm{Hz}$. After MESNa treatment to remove noninternalized biotin, cells were fixed and labeled with antibodies against caveolin 1 and cavin 1 , and with fluorescent streptavidin. Bars, 10 $\mu m$. (D) Quantification of biotin uptake after surface labeling with sulfo-NHS-SS-biotin and MESNa treatment. Mean fluorescence intensity of multiple cell regions after subtraction of background signal is shown. (E) Quantification of the proportion of caveolin 1 present in biotin-positive (i.e., endosomal) pixels after incubation as in $C$. mine-treated animals (Fig. 4, A and B). Electron microscopy of cardiac muscle in control and caveolin $1^{-1-}$ animals stimulated with dobutamine revealed that ruptured endothelial cells and discontinuities in the endothelial layer could be readily detected (Fig. 4 C). Together, these data provide direct evidence that the plasma membrane of caveolin $1^{-/-}$endothelial cells is less able to withstand the increased hemodynamic forces caused by increases in cardiac output.

One of the cardiovascular phenotypes of caveolin $1^{-/-}$ mice is that hypoxia induces heart failure (Cruz et al., 2012; Mathew, 2014). Because caveolin 1 is not expressed in cardiac muscle, one would predict that this could be a result of endothelial cell dysfunction. Given our findings, it seemed likely that ruptured endothelial cells will contribute to the phenotype. To test this, electron microscopy was used to examine the ultrastructure of endothelial cells from microvessels in cardiac muscle of the right ventricle of mice exposed to hypoxic conditions for $3 \mathrm{wk}$. Changes in endothelial morphology were strikingly apparent in the caveolin $1^{-/-}$animals (Fig. 5 A). Endothelial cells that appeared devoid of cytoplasmic staining, and had a distended membrane outline, were frequently detected (Fig. 5 A). Quantification of the abundance of these abnormal 
A
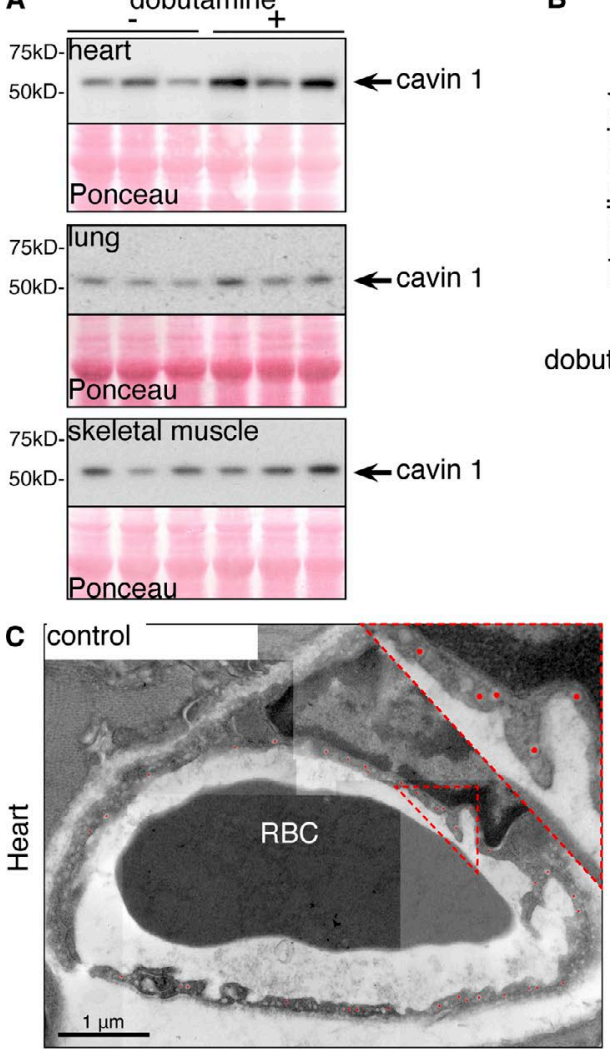

D control

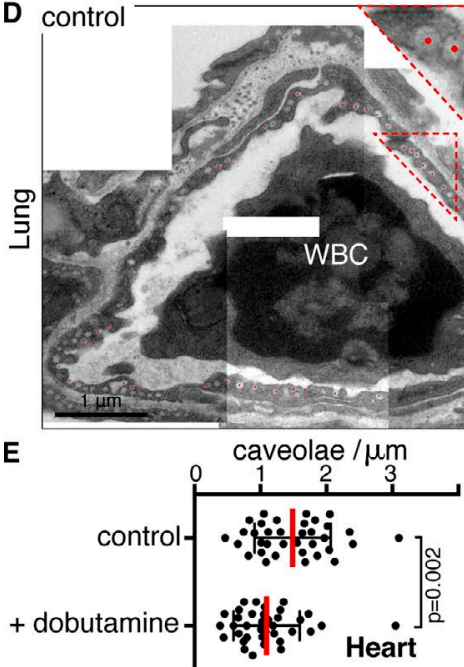

B

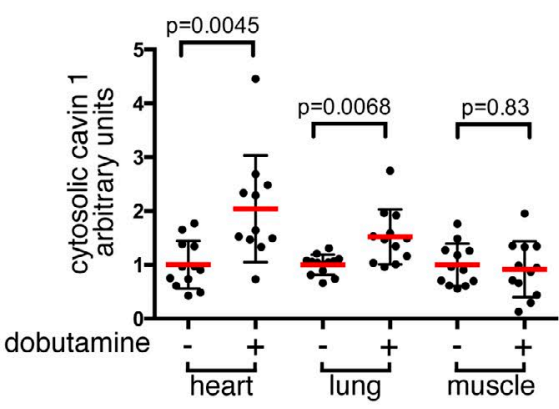

Figure 3. Increased cardiac output causes caveolar disassembly in vivo. (A) Immunoblot analyses of cavin-1 in cytosolic fractions from tissues snap frozen after $1 \mathrm{~min}$ of treatment of mice with dobutamine. Samples from three separate animals are shown for each tissue/ treatment. Ponceau stain of the relevant Western blots is also included, to confirm equal loading. (B) Quantification of immunoblots of cytosolic cavin 1 as in A. Densitometry data in arbitrary units were normalized such that the mean of the control (i.e., without dobutamine) for each tissue is equal to 1 . Each point represents one animal; mice were 20-25 wk old. (C) Multiple electron micrographs to represent endothelium of microvessels from the heart of control and dobutamine-treated mice. Red dots indicate structures counted as caveolae open to the luminal side of the endothelial cell. Dobutamine treatment was for 1 min before rapid dissection and tissue fixation. Zoomed-in regions are shown as outlined. (D) Electron micrographs of microvessels from the lung of control and dobutamine-treated mice, as in C. (E) Quantification of caveolae in microvascular endothelium of the heart. Dobutamine treatment was for $1 \mathrm{~min}$. Each point represents one complete reconstruction of the endothelium from multiple micrographs as in $C$; data are from three control and three treated mice. (F) As in E, but tissue is lung. RBC and WBC denote red and white blood cells, respectively.

endothelial cells confirmed that caveolae play a significant role in preventing cell damage during hypoxia (Fig. 5 B). We suggest that endothelial cell dysfunction underlies cardiovascular phenotypes of caveolin $1^{-/-}$mice, and that rupture or damage to the plasma membrane of endothelial cells is likely to contribute to these phenotypes.

\section{Discussion}

Two different but nonexclusive mechanisms have been proposed that could explain the mechanoprotective role for caveolae in vivo revealed by our experiments. Caveolae can disassemble, and flattening of the membrane of the caveolar bulb would then increase the effective surface area of the cell, preventing buildup of excessive force within the plane of the membrane (Sinha et al., 2011). Additionally, caveolae may bud from the membrane and internalize damaged regions, leading to resealing (Corrotte et al., 2013). Counting of caveolae in the plasma membrane does not discriminate between the two models because both flattening and budding may decrease the number of surface-connected caveolae. Our in vivo experiments show that cavin 1 is released from the membrane to cytosolic fractions upon increased blood flow. Given the considerable ex vivo data suggesting that association of cavin 1 with caveolin 1 at the plasma membrane is key for adoption of the characteristic membrane shape of caveolae (Hill et al., 2008; Liu and Pilch, 2008; Hayer et al., 2010; Ludwig et al., 2013; Gambin et al., 2014), and our biochemical analysis of disassembly of the caveolar coat complex under conditions where endocytosis is 
A
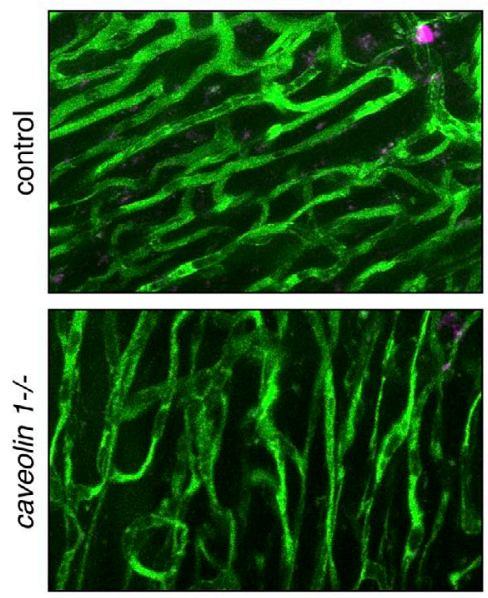

B

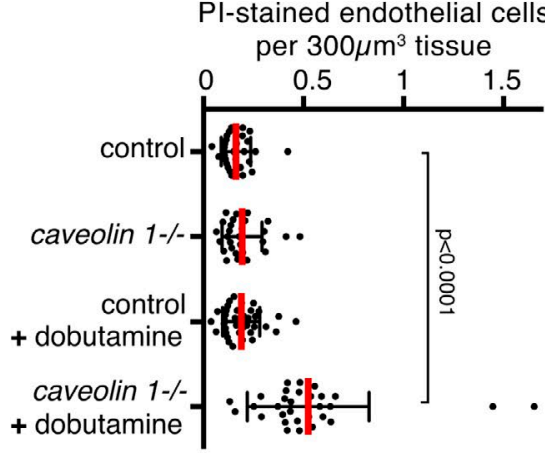

+ dobutamine

Pl-stained endothelial cells
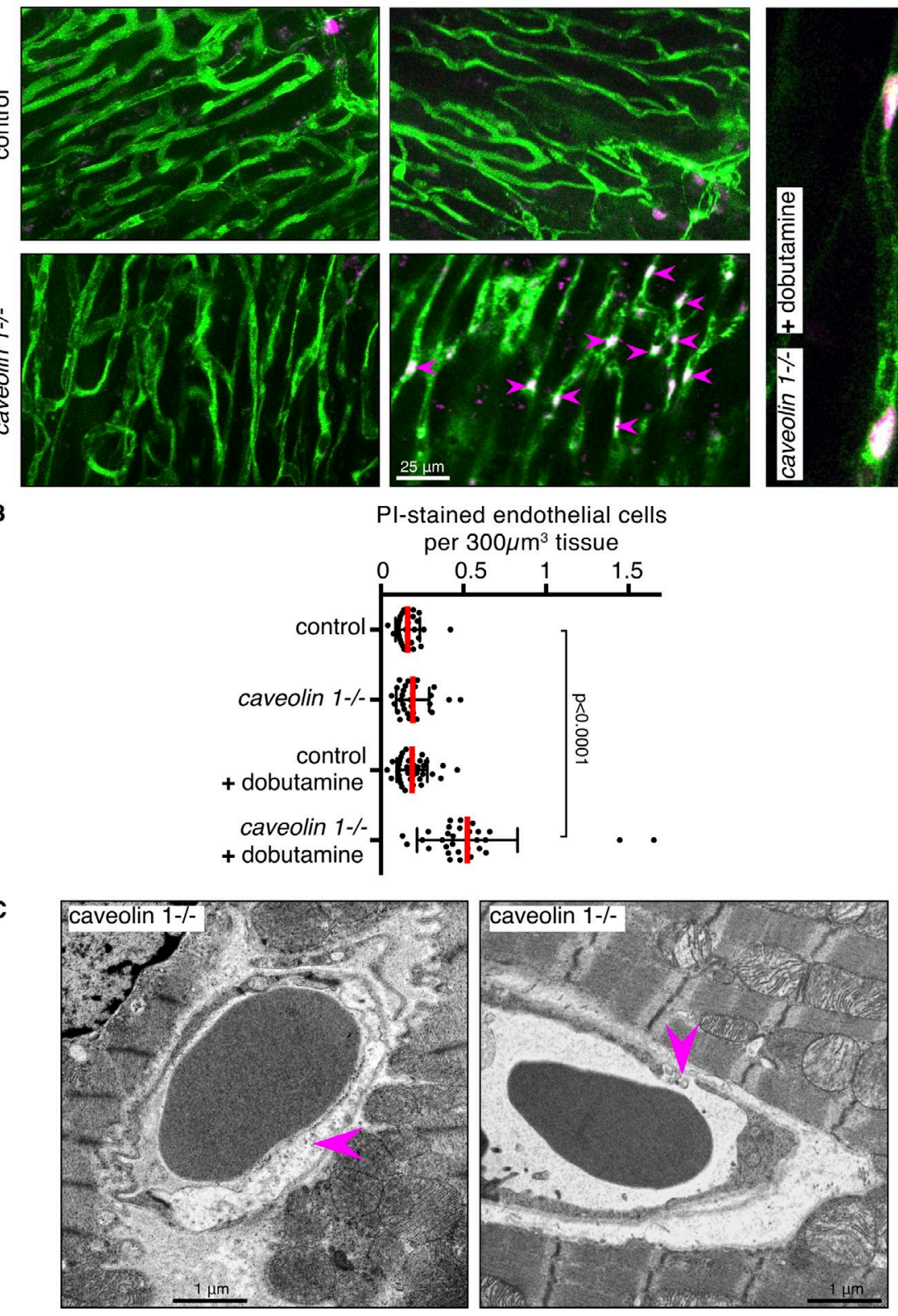

Figure 4. Endothelial caveolae protect cells from membrane damage during acute increases in cardiac output in vivo. (A) Maximum intensity projections of $z$ stacks of two-photon images ( 20 $\mu \mathrm{m}$ deep) of heart tissue from control and caveolin 1-/- mice injected with FITC-albumin (green, capillaries) and propidium iodide ( $\mathrm{Pl}$; magenta, nuclei), with and without injection of dobutamine. Arrows show Pl-positive nuclei within capillaries. The zoomed-in view in the right-hand panel confirms that Pl-positive nuclei are in capillaries. (B) Quantification of Pl-positive nuclei within capillaries. Each point represents one image stack, greater than five animals were analyzed per condition. (C) Abnormal endothelial morphology in cardiac muscle of caveolin 1-/mice immediately after dobutamine treatment. Examples of ruptured cells and of abnormal gaps between cells are arrowed. blocked in ex vivo experiments (Figs. 1 and 2), our data then provide strong support for the caveolae flattening model.

There is considerable evidence for endothelial cell dysfunction and cardiovascular phenotypes in mice lacking caveolin 1; however, these phenotypes do not by themselves lend strong support for one specific mechanism by which caveolae act (Drab et al., 2001; Schubert et al., 2002; Zhao et al., 2002; Chang et al., 2009; Rajab et al., 2010; Siddiqui et al., 2011; Austin et al., 2012; Cruz et al., 2012). The accumulation of damaged endothelial cells under chronic conditions, such as hypoxia in our experiments, could be attributable to many factors involving cell signaling or other processes. However, our data show that by using an acute perturbation ( 1 min of dobutamine treatment) and an assay, which specifically detects loss of plasma membrane integrity, that loss of caveolae leads to instantaneous rupture of the membrane under increased hemodynamic stress. We suggest that this mechanism is likely to be a contributing factor to the range of cardiovascular phenotypes consequent to deletion of caveolin 1.
Although disassembly of caveolae to release membrane convolutions, and thereby buffer mechanical force within the plasma membrane, is an appealingly simple mechanism by which caveolae may exert mechanoprotective effects, and although this mechanism provides an elegant rationale for the extreme abundance of caveolae within endothelium, it is possible that caveolae may have further functional roles. Of note, mechanical insults to cells trigger phosphorylation of caveolin 1 on Tyrosine 14, and readout of this phosphorylation by cellular signaling factors may have multiple effects that remain to be fully determined (Volonté et al., 2001; del Pozo et al., 2005; Joshi et al., 2012).

Repetitive membrane damage as a result of mechanical force has been well studied in the context of muscle cells and other systems (Lammerding and Lee, 2007). Similar damage to endothelial cells has been less investigated; however, studies in other systems suggest multiple mechanisms that may allow cells to reseal damaged plasma membrane 

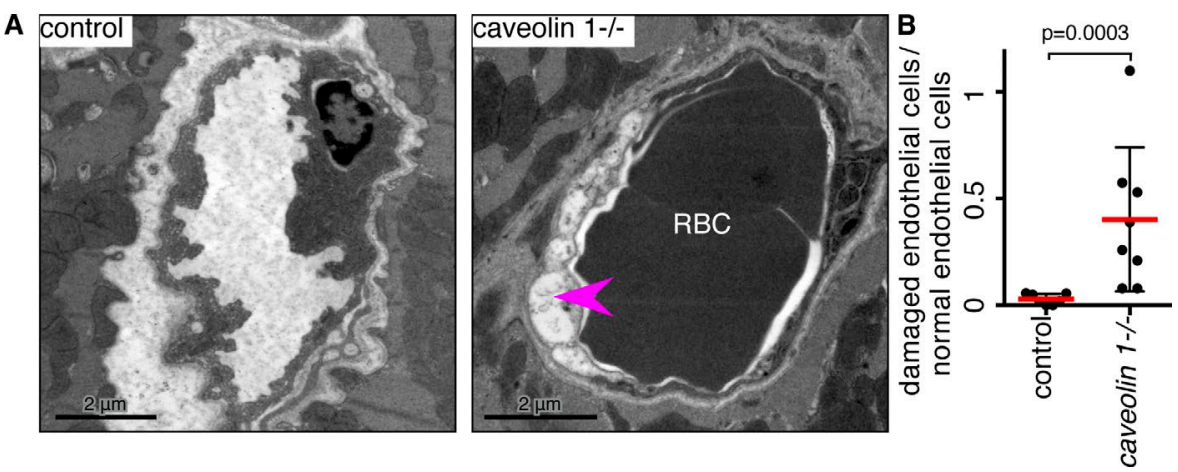

Figure 5. Endothelial caveolae protect cells from membrane damage during hypoxia in vivo. (A) Electron micrographs of microvessels from the right ventricle of heart from mice exposed to hypoxia for 3 wk. Magenta arrowhead highlights an abnormal endothelial cell with loss of cytoplasmic staining. (B) Quantification of endothelial cells with abnormal morphology after 3 wk hypoxia. Each dot represents one animal, $>20$ vessels were examined for each animal.
(Lammerding and Lee, 2007; Han, 2011; Jimenez et al., 2014). It is probable that these or similar mechanisms act in endothelial cells, thus flattening of caveolae will provide one layer of a complex set of processes involved in mechanoprotection. However, disassembly of caveolae is conceptually different from mechanisms for resealing damaged plasma membrane because it provides the cell with a method to prevent lesions rather than to repair them.

\section{Materials and methods}

\section{Animal procedures}

All experiments using mice were conducted under the appropriate UK Home Office license, and were approved by the Ethical Review Committee of the Medical Research Council, Laboratory of Molecular Biology, or of the University of Cambridge. Caveolin $1^{-/-}$mice have the first two exons of the $C A V 1$ gene deleted (Razani et al., 2001). We have backcrossed these mice onto the C57BL/6J background for more than five generations (Hansen et al., 2013).

\section{Antibodies and reagents}

The following antibodies were used: rabbit anti-cavin 1 (ab48824; Abcam) and rabbit anti-caveolin 1 (610060; BD Biosciences). HRP-conjugated secondary antibodies were from DAKO and fluorophore-conjugated antibodies and streptavidin were from Molecular Probes (Invitrogen). Further reagents include FITC-albumin (A9771; Sigma-Aldrich), Propidium Iodide solution (P4864; Sigma-Aldrich), and dobutamine (Hameln Pharmaceuticals).

\section{Cytosol and membrane extraction-differential centrifugation}

Cells were lysed in $10 \mathrm{mM}$ Tris/Cl pH 7.5, $150 \mathrm{mM} \mathrm{NaCl}$, and $0.5 \mathrm{mM}$ EDTA without detergent by using a $25-\mathrm{G}$ needle for 100 strokes. Cell lysates were spun at $13,500 \mathrm{rpm}$ for $10 \mathrm{~min}$ to remove large cellular debris followed by a final centrifugation step at 55,000 rpm in a benchtop Optima Max ultracentrifuge (Beckman Coulter) to isolate membrane and cytosolic fractions. For analysis of tissues from mice, the animals were killed by cervical dislocation. Immediately, and as rapidly as possible (within $30 \mathrm{~s}$ of cervical dislocation), the heart, lungs, and abdominal muscle were removed in a defined order and instantly frozen in liquid nitrogen. Frozen tissues were ground using a pestle and mortar before differential centrifugation as described previously.

\section{Sucrose velocity gradients}

Chemical cross-linking was performed precisely as described (Ludwig et al., 2013). Either cytosolic extracts or cross-linked whole cell lysates in $1 \%$ (vol/vol) Triton X-100, $2 \%$ (wt/vol) octyl-glucoside were added atop a linear $10-40 \%(\mathrm{wt} / \mathrm{vol})$ sucrose gradient prepared in $50 \mathrm{mM}$ Tris, $\mathrm{pH} 8,300 \mathrm{mM} \mathrm{NaCl}, 5 \mathrm{mM}$ EDTA, and protease inhibitor cocktail (Roche), plus $0.2 \%$ Triton X-100 for the detergent-solubilized lysates. Gradients were spun in a SW 40 Ti Rotor (Beckman Coulter) at $37,000 \mathrm{rpm}$ for $6 \mathrm{~h}$ at $4^{\circ} \mathrm{C}$. Twelve $1-\mathrm{ml}$ fractions were collected from the bottom of the gradient by tube puncture (Ludwig et al., 2013).

\section{Hypo-osmotic medium}

Cells were incubated in hypo-osmotic medium containing a 1:9 ratio of DMEM with $10 \% \mathrm{FBS} /$ water.

\section{Mechanical stretching}

A ShellPa mechanical cell stretcher (B-Bridge International/MetaChem Diagnostics) was used at a frequency of $1 \mathrm{cps}$ at $20 \%$ stretch ratio within a $37^{\circ} \mathrm{C}$ incubator to stretch bEnd5 cells grown in fibronectin-coated (Sigma-Aldrich) chambers made from polydimethylsiloxane.

\section{Two-photon imaging and in vivo experiments}

Mice were injected intravenously with $200 \mu \mathrm{l}$ of $25 \mathrm{mg} / \mathrm{ml}$ albumin conjugated to FITC (Sigma-Aldrich). Where heart stimulation was required, dobutamine was used at $30 \mu \mathrm{g} / \mathrm{g}$ intraperitoneally. For assessment of damaged endothelial cells in vivo, propidium iodide was introduced intravenously at $2 \mathrm{mg} / \mathrm{kg}$. Heart rates before and after dobutamine injections were monitored using a MouseOx Plus pulse oximeter (Starr Life Sciences Corp.). Heart and abdominal muscle were dissected for two-photon imaging of nonfixed, fresh wholemount tissues using a 710 NLO upright microscope (Carl Zeiss). A 20×, W Plan-Apochromat 20× 1.2 NA water immersion objective was used to acquire all images. Dissected tissue was immersed in PBS and imaged at RT.

\section{Confocal imaging}

All confocal imaging was performed using an LSM 780 inverted confocal microscope (Carl Zeiss) with a 63×, 1.4 NA objective, driven by Zen software (Carl Zeiss). Quantification of image overlap was performed as described (Bitsikas et al., 2014).

\section{Image processing}

Images were handled with Adobe Photoshop and ImageJ software. Contrast settings were adjusted to facilitate visualization of overlaid pseudo-color images. All such manipulations were applied equally across the whole image. Where indicated, maximum intensity projections of multiple planes in $\mathrm{z}$ stacks were calculated using ImageJ.

\section{Electron microscopy}

Mouse tissue was dissected out and fixed in $2.5 \%$ glutaraldehyde $2 \%$ PFA in $0.1 \mathrm{M}$ Cacodylate buffer, $\mathrm{pH}$ 7.4. After washing, tissue was post fixed in $1 \%$ osmium tetroxide, dehydrated in an ascending ethanol series followed by propylene oxide, and infiltrated and embedded in $\mathrm{CY} 212$ resin. 
bEnd5 cells grown on MatTek glass-bottomed Petri dishes, subjected to hypo-osmotic medium as described, were fixed in $2.5 \%$ glutaraldehyde 2\% PFA in $0.1 \mathrm{M}$ Cacodylate buffer, as were bEnd5 cells grown on fibronectin-coated silicon chambers after stretching. Cells were then processed on their growing substrate for electron microscopy as described previously but without propylene oxide. Cells were cut perpendicular to their growing substrate and sections of cells and tissue were stained with saturated aqueous uranyl acetate and Reynolds lead citrate and viewed at $80 \mathrm{kV}$ on a Spirit or T12 TEM (FEI). Quantification of caveolae in endothelium was performed by acquiring images to trace the outline of microvessels at 6,500 magnification, assembling these images into complete profiles of the vessel using Adobe Photoshop, and then scoring morphological caveolae blind to the identity of the sample.

\section{Analysis of SYTOX Green uptake in cell lines and membrane rupture assay}

Mouse embryonic fibroblasts were incubated in hypotonic medium for up to $1 \mathrm{~h}$ to induce membrane rupture. SYTOX Green (Life Technologies), a cell membrane-impermeant nucleic acid dye, was used at 0.5 $\mathrm{nM}$ for uptake into damaged cells. Cells were trypsinized after hypotonic shock and resuspended in PBS containing 10\% FBS to measure SYTOX Green signal using an iCyt Eclipse (EC800) flow cytometer (Sony). Cells were not trypsinized before flow cytometry analysis because trypsinization affects caveolar complex stability (del Pozo et al., 2005). Time-lapse imaging was performed at $1 \mathrm{~min}$ intervals in $\mathrm{CO}_{2}$-independent media.

\section{Hypoxia}

The 16-wk-old mice were placed in a hypoxic chamber $\left(10 \% \mathrm{O}_{2}\right.$, $21^{\circ} \mathrm{C} \pm 2{ }^{\circ} \mathrm{C}$, and $55 \% \pm 10 \%$ ) for 3 wk before tissue dissection for electron microscopy.

\section{Statistical analysis}

All p-values are from a Mann-Whitney test. All bars in graphs represent the mean and SD from the mean.

\section{Online supplemental material}

Fig. S1 presents quantification of abundance of caveolae by electron microscopy in mechanically stretched cells. Fig. S2 presents additional data to support the assay of caveolar disassembly in dobutamine-treated mice shown in Fig. 3. Fig. S3 presents assay of plasma membrane rupture in tissue culture cells. Online supplemental material is available at http://www.jcb.org/cgi/content/ full/jcb.201504042/DC1.

\section{Acknowledgments}

We thank Claire Knox, Richard Berks, and other staff members at Medical Research Council (MRC) Ares for expert technical support. Cristina Branco-Price provided much appreciated assistance with i.v. injections.

N.W. Morrell, B.J. Dunmore, and A. Crosby were funded by the British Heart Foundation. E. Shvets was funded by an EU Marie Curie Fellowship. C. Mendoza-Topaz was funded by the British Heart Foundation. All other funding was from the UK MRC.

The authors declare no competing financial interests.

Submitted: 9 April 2015

Accepted: 2 September 2015

\section{References}

Albinsson, S., I. Nordström, K. Swärd, and P. Hellstrand. 2008. Differential dependence of stretch and shear stress signaling on caveolin-1 in the vascular wall. Am. J. Physiol. Cell Physiol. 294:C271-C279. http://dx.doi. org/10.1152/ajpcell.00297.2007

Andrews, N.W., P.E. Almeida, and M. Corrotte. 2014. Damage control: cellular mechanisms of plasma membrane repair. Trends Cell Biol. 24:734-742. http://dx.doi.org/10.1016/j.tcb.2014.07.008

Ardissone, A., C. Bragato, L. Caffi, F. Blasevich, S. Maestrini, M.L. Bianchi, L. Morandi, I. Moroni, and M. Mora. 2013. Novel PTRF mutation in a child with mild myopathy and very mild congenital lipodystrophy. BMC Med. Genet. 14:89. http://dx.doi.org/10.1186/1471-2350-14-89

Austin, E.D., L. Ma, C. LeDuc, E. Berman Rosenzweig, A. Borczuk, J.A. Phillips III, T. Palomero, P. Sumazin, H.R. Kim, M.H. Talati, et al. 2012. Whole exome sequencing to identify a novel gene (caveolin-1) associated with human pulmonary arterial hypertension. Circ Cardiovasc Genet. 5:336343. http://dx.doi.org/10.1161/CIRCGENETICS.111.961888

Bitsikas, V., I.R. Corrêa Jr., and B.J. Nichols. 2014. Clathrin-independent pathways do not contribute significantly to endocytic flux. eLife. 3:e03970.

Chang, S.H., D. Feng, J.A. Nagy, T.E. Sciuto, A.M. Dvorak, and H.F. Dvorak. 2009. Vascular permeability and pathological angiogenesis in caveolin-1-null mice. Am. J. Pathol. 175:1768-1776. http://dx.doi.org/10.2353/ ajpath.2009.090171

Collins, B.M., M.J. Davis, J.F. Hancock, and R.G. Parton. 2012. Structure-based reassessment of the caveolin signaling model: do caveolae regulate signaling through caveolin-protein interactions? Dev. Cell. 23:11-20. http:// dx.doi.org/10.1016/j.devcel.2012.06.012

Corrotte, M., P.E. Almeida, C. Tam, T. Castro-Gomes, M.C. Fernandes, B.A. Millis, M. Cortez, H. Miller, W. Song, T.K. Maugel, et al. 2013. Caveolae internalization repairs wounded cells and muscle fibers. eLife. 2:e00926. http://dx.doi.org/10.7554/eLife.00926

Cruz, J.A., E.M. Bauer, A.I. Rodriguez, A. Gangopadhyay, N.S. Zeineh, Y. Wang, S. Shiva, H.C. Champion, and P.M. Bauer. 2012. Chronic hypoxia induces right heart failure in caveolin-1-/- mice. Am. J. Physiol. Heart Circ. Physiol. 302:H2518-H2527. http://dx.doi.org/10.1152/ ajpheart.01140.2011

del Pozo, M.A., N. Balasubramanian, N.B. Alderson, W.B. Kiosses, A. GrandeGarcía, R.G. Anderson, and M.A. Schwartz. 2005. Phospho-caveolin-1 mediates integrin-regulated membrane domain internalization. Nat. Cell Biol. 7:901-908. http://dx.doi.org/10.1038/ncb1293

Desai, K.H., R. Sato, E. Schauble, G.S. Barsh, B.K. Kobilka, and D. Bernstein. 1997. Cardiovascular indexes in the mouse at rest and with exercise: new tools to study models of cardiac disease. Am. J. Physiol. 272:H1053-H1061.

Drab, M., P. Verkade, M. Elger, M. Kasper, M. Lohn, B. Lauterbach, J. Menne, C. Lindschau, F. Mende, F.C. Luft, et al. 2001. Loss of caveolae, vascular dysfunction, and pulmonary defects in caveolin-1 gene-disrupted mice. Science. 293:2449-2452. http://dx.doi.org/10.1126/science.1062688

Dulhunty, A.F., and C. Franzini-Armstrong. 1975. The relative contributions of the folds and caveolae to the surface membrane of frog skeletal muscle fibres at different sarcomere lengths. J. Physiol. 250:513-539. http:// dx.doi.org/10.1113/jphysiol.1975.sp011068

Galbiati, F., J.A. Engelman, D. Volonte, X.L. Zhang, C. Minetti, M. Li, H. Hou Jr., B. Kneitz, W. Edelmann, and M.P. Lisanti. 2001a. Caveolin-3 null mice show a loss of caveolae, changes in the microdomain distribution of the dystrophin-glycoprotein complex, and T-tubule abnormalities. J. Biol. Chem. 276:21425-21433. http://dx.doi.org/10.1074/jbc.M100828200

Galbiati, F., B. Razani, and M.P. Lisanti. 2001b. Caveolae and caveolin-3 in muscular dystrophy. Trends Mol. Med. 7:435-441. http://dx.doi.org/10.1016/ S1471-4914(01)02105-0

Gambin, Y., N. Ariotti, K.A. McMahon, M. Bastiani, E. Sierecki, O. Kovtun, M.E. Polinkovsky, A. Magenau, W. Jung, S. Okano, et al. 2014. Singlemolecule analysis reveals self assembly and nanoscale segregation of two distinct cavin subcomplexes on caveolae. eLife. 3:e01434. http://dx.doi. org/10.7554/eLife.01434

García-Cardeña, G., P. Oh, J. Liu, J.E. Schnitzer, and W.C. Sessa. 1996. Targeting of nitric oxide synthase to endothelial cell caveolae via palmitoylation: implications for nitric oxide signaling. Proc. Natl. Acad. Sci. USA. 93:6448-6453. http://dx.doi.org/10.1073/pnas.93.13.6448

Han, R. 2011. Muscle membrane repair and inflammatory attack in dysferlinopathy. Skelet. Muscle. 1:10.

Hansen, C.G., E. Shvets, G. Howard, K. Riento, and B.J. Nichols. 2013. Deletion of cavin genes reveals tissue-specific mechanisms for morphogenesis of endothelial caveolae. Nat. Commun. 4:1831. http://dx.doi.org/10.1038/ ncomms 2808 
Hayer, A., M. Stoeber, C. Bissig, and A. Helenius. 2010. Biogenesis of caveolae: stepwise assembly of large caveolin and cavin complexes. Traffic. 11:361-382. http://dx.doi.org/10.1111/j.1600-0854.2009.01023.x

Hill, M.M., M. Bastiani, R. Luetterforst, M. Kirkham, A. Kirkham, S.J. Nixon, P. Walser, D. Abankwa, V.M. Oorschot, S. Martin, et al. 2008. PTRFCavin, a conserved cytoplasmic protein required for caveola formation and function. Cell. 132:113-124. http://dx.doi.org/10.1016/j. cell.2007.11.042

Jimenez, A.J., P. Maiuri, J. Lafaurie-Janvore, S. Divoux, M. Piel, and F. Perez. 2014. ESCRT machinery is required for plasma membrane repair. Science. 343:1247136. http://dx.doi.org/10.1126/science.1247136

Jones, L.J., and V.L. Singer. 2001. Fluorescence microplate-based assay for tumor necrosis factor activity using SYTOX Green stain. Anal. Biochem. 293:8-15. http://dx.doi.org/10.1006/abio.2001.5116

Joshi, B., M. Bastiani, S.S. Strugnell, C. Boscher, R.G. Parton, and I.R. Nabi. 2012. Phosphocaveolin-1 is a mechanotransducer that induces caveola biogenesis via Egr1 transcriptional regulation. J. Cell Biol. 199:425-435. http://dx.doi.org/10.1083/jcb.201207089

Lammerding, J., and R.T. Lee. 2007. Torn apart: membrane rupture in muscular dystrophies and associated cardiomyopathies. J. Clin. Invest. 117:17491752. http://dx.doi.org/10.1172/JCI32686

Lee, J., and G.W. Schmid-Schönbein. 1995. Biomechanics of skeletal muscle capillaries: hemodynamic resistance, endothelial distensibility, and pseudopod formation. Ann. Biomed. Eng. 23:226-246. http://dx.doi. org/10.1007/BF02584425

Liu, L., and P.F. Pilch. 2008. A critical role of cavin (polymerase I and transcript release factor) in caveolae formation and organization. J. Biol. Chem. 283:4314-4322. http://dx.doi.org/10.1074/jbc.M707890200

Liu, L., D. Brown, M. McKee, N.K. Lebrasseur, D. Yang, K.H. Albrecht, K. Ravid, and P.F. Pilch. 2008. Deletion of Cavin/PTRF causes global loss of caveolae, dyslipidemia, and glucose intolerance. Cell Metab. 8:310-317. http://dx.doi.org/10.1016/j.cmet.2008.07.008

Ludwig, A., G. Howard, C. Mendoza-Topaz, T. Deerinck, M. Mackey, S. Sandin, M.H. Ellisman, and B.J. Nichols. 2013. Molecular composition and ultrastructure of the caveolar coat complex. PLoS Biol. 11:e1001640. http:// dx.doi.org/10.1371/journal.pbio. 1001640

Marcovitz, P.A., and W.F. Armstrong. 1991. Dobutamine stress echocardiography: diagnostic utility. Herz. 16:372-378.

Mathew, R. 2014. Pathogenesis of pulmonary hypertension: a case for caveolin-1 and cell membrane integrity. Am. J. Physiol. Heart Circ. Physiol. 306:H15-H25.

Oh, P., P. Borgström, H. Witkiewicz, Y. Li, B.J. Borgström, A. Chrastina, K. Iwata, K.R. Zinn, R. Baldwin, J.E. Testa, et al. 2007. Live dynamic imaging of caveolae pumping targeted antibody rapidly and specifically across endothelium in the lung. Nat. Biotechnol. 25:327-337. http:// dx.doi.org/10.1038/nbt1292

Parker, S., H.S. Peterkin, and H.A. Baylis. 2007. Muscular dystrophy associated mutations in caveolin-1 induce neurotransmission and locomotion defects in Caenorhabditis elegans. Invert. Neurosci. 7:157-164. http:// dx.doi.org/10.1007/s10158-007-0051-5

Parton, R.G., and M.A. del Pozo. 2013. Caveolae as plasma membrane sensors, protectors and organizers. Nat. Rev. Mol. Cell Biol. 14:98-112. http:// dx.doi.org/10.1038/nrm3512
Parton, R.G., and K. Simons. 2007. The multiple faces of caveolae. Nat. Rev. Mol. Cell Biol. 8:185-194. http://dx.doi.org/10.1038/nrm2122

Pilch, P.F., and L. Liu. 2011. Fat caves: caveolae, lipid trafficking and lipid metabolism in adipocytes. Trends Endocrinol. Metab. 22:318-324. http:// dx.doi.org/10.1016/j.tem.2011.04.001

Predescu, S.A., D.N. Predescu, and A.B. Malik. 2007. Molecular determinants of endothelial transcytosis and their role in endothelial permeability. Am. J. Physiol. Lung Cell. Mol. Physiol. 293:L823-L842. http://dx.doi. org/10.1152/ajplung.00436.2006

Rajab, A., V. Straub, L.J. McCann, D. Seelow, R. Varon, R. Barresi, A. Schulze, B. Lucke, S. Lützkendorf, M. Karbasiyan, et al. 2010. Fatal cardiac arrhythmia and long-QT syndrome in a new form of congenital generalized lipodystrophy with muscle rippling (CGL4) due to PTRF-CAVIN mutations. PLoS Genet. 6:e1000874. http://dx.doi.org/10.1371/journal. pgen. 1000874

Razani, B., J.A. Engelman, X.B. Wang, W. Schubert, X.L. Zhang, C.B. Marks, F. Macaluso, R.G. Russell, M. Li, R.G. Pestell, et al. 2001. Caveolin-1 null mice are viable but show evidence of hyperproliferative and vascular abnormalities. J. Biol. Chem. 276:38121-38138.

Schubert, W., P.G. Frank, S.E. Woodman, H. Hyogo, D.E. Cohen, C.W. Chow, and M.P. Lisanti. 2002. Microvascular hyperpermeability in caveolin-1 (-/-) knock-out mice. Treatment with a specific nitric-oxide synthase inhibitor, L-NAME, restores normal microvascular permeability in Cav-1 null mice. J. Biol. Chem. 277:40091-40098. http://dx.doi.org/10.1074/ jbc.M205948200

Shvets, E., V. Bitsikas, G. Howard, C.G. Hansen, and B.J. Nichols. 2015. Dynamic caveolae exclude bulk membrane proteins and are required for sorting of excess glycosphingolipids. Nat. Commun. 6:6867. http:// dx.doi.org/10.1038/ncomms 7867

Siddiqui, M.R., Y.A. Komarova, S.M. Vogel, X. Gao, M.G. Bonini, J. Rajasingh, Y.Y. Zhao, V. Brovkovych, and A.B. Malik. 2011. Caveolin-1-eNOS signaling promotes p190RhoGAP-A nitration and endothelial permeability. J. Cell Biol. 193:841-850. http://dx.doi.org/10.1083/jcb.201012129

Sinha, B., D. Köster, R. Ruez, P. Gonnord, M. Bastiani, D. Abankwa, R.V. Stan, G. Butler-Browne, B. Vedie, L. Johannes, et al. 2011. Cells respond to mechanical stress by rapid disassembly of caveolae. Cell. 144:402-413. http://dx.doi.org/10.1016/j.cell.2010.12.031

Tse, D., and R.V. Stan. 2010. Morphological heterogeneity of endothelium. Semin. Thromb. Hemost. 36:236-245. http://dx.doi.org/10.1055/s-0030-1253447

Volonté, D., F. Galbiati, R.G. Pestell, and M.P. Lisanti. 2001. Cellular stress induces the tyrosine phosphorylation of caveolin-1 $\left(\mathrm{Tyr}^{14}\right)$ via activation of p38 mitogen-activated protein kinase and c-Src kinase. Evidence for caveolae, the actin cytoskeleton, and focal adhesions as mechanical sensors of osmotic stress. J. Biol. Chem. 276:8094-8103. http://dx.doi. org/10.1074/jbc.M009245200

Woodman, S.E., F. Sotgia, F. Galbiati, C. Minetti, and M.P. Lisanti. 2004 Caveolinopathies: mutations in caveolin-3 cause four distinct autosomal dominant muscle diseases. Neurology. 62:538-543. http://dx.doi. org/10.1212/WNL.62.4.538

Zhao, Y.Y., Y. Liu, R.V. Stan, L. Fan, Y. Gu, N. Dalton, P.H. Chu, K. Peterson, J. Ross Jr., and K.R. Chien. 2002. Defects in caveolin-1 cause dilated cardiomyopathy and pulmonary hypertension in knockout mice. Proc. Natl. Acad. Sci. USA. 99:11375-11380. http://dx.doi.org/10.1073/ pnas. 172360799 